OPEN ACCESS

Edited by:

Karel Allegaert,

University Hospitals Leuven, Belgium

Reviewed by:

Bruno Martinez-Leo, Universidad Nacional Autónoma de

México, Mexico

Tuuli Metsvaht

University of Tartu, Estonia

*Correspondence:

Ming-Chou Chiang

newborntw@gmail.com

Specialty section: This article was submitted to Neonatology,

a section of the journa

Frontiers in Pediatrics

Received: 11 October 2020 Accepted: 04 January 2021 Published: 25 January 2021

Citation:

Chang H-P, Fu R-H, Lin J-J and Chiang M-C (2021) Prognostic Factors and Clinical Features of Neonatal Splenic

Rupture/Hemorrhage: Two Cases

Reports and Literature Review.

Front. Pediatr. 9:616247.

doi: 10.3389/fped.2021.616247

\section{Prognostic Factors and Clinical Features of Neonatal Splenic Rupture/Hemorrhage: Two Cases Reports and Literature Review}

\author{
Han-Pi Chang ${ }^{1,2}$, Ren-Huei Fu ${ }^{1}$, Jainn-Jim Lin ${ }^{2,3}$ and Ming-Chou Chiang ${ }^{1,3 *}$ \\ 'Division of Neonatology, Department of Pediatrics, Chang Gung Memorial Hospital and Chang Gung University College of \\ Medicine, Taoyuan, Taiwan, ${ }^{2}$ Division of Pediatric Critical Care Medicine, Department of Pediatrics, Chang Gung Memorial \\ Hospital and Chang Gung University College of Medicine, Taoyuan, Taiwan, ${ }^{3}$ Graduate Institute of Clinical Medical Sciences, \\ Chang Gung University College of Medicine, Taoyuan, Taiwan
}

Background: Neonatal splenic rupture/hemorrhage (SRH), an extremely rare and potentially fatal presentation, can spontaneously resolve without surgical treatment; However, treatment approaches remain controversial. The present study aimed to describe and analyze the clinical features and therapies of neonatal $\mathrm{SRH}$ and therapeutic approaches.

Methods: We present the cases of two patients and review another 37 cases reported in English-literature. The literature search included all articles published in PUBMED from inception between January 1968 and December 2019. Demographic data, precipitating factors, clinical characteristics including presenting symptoms and signs, presenting time, age at $\mathrm{SRH}$ presentation, imaging findings, as well as treatments and outcomes were analyzed.

Results: In addition to the two cases treated at our hospital, 37 neonates with $\mathrm{SRH}$ were reported during the study period. The rate of full-term neonates was $72 \%$ (28/39). The cause was idiopathic in most cases, and congenital coagulation disorders were underlying causes in $13 \%(5 / 39)$ of the cases. The most common presenting symptom and sign of neonatal $\mathrm{SRH}$ were pallor or anemia, followed by abdominal discoloration/distension. Additionally, 18\% (7/39) of the cases presented with scrotal hematoma or swelling. The age at $\mathrm{SRH}$ presentation ranged between $3 \mathrm{~h}$ and 5 days of age. Abdominal ultrasonography or computed tomography was used as the diagnostic tool. Twenty-seven cases (69\%) received surgical management. The prognosis was comparable between the neonates treated with splenectomy and those treated with non-surgical approaches. The mortality rate was 18\% (7/39) in the study cohort. SRH presentation at $\leq 12 \mathrm{~h}$ of age was associated with higher mortality compared to $\mathrm{SRH}$ presenting time at $>12 \mathrm{~h}$ of age (odds ratio $25.0,95 \% \mathrm{Cl} 2.514-248.575, p=0.001$ ).

Conclusion: Our literature review revealed that the mortality rate of neonatal $\mathrm{SRH}$ was $18 \%$ and that the mortality risk was higher in neonates presenting with $\mathrm{SRH}$ symptoms and signs at $\leq 12 \mathrm{~h}$ of age.

Keywords: neonates, spleen, splenic hematoma, splenic hemorrhage, splenic laceration, splenic rupture 


\section{INTRODUCTION}

Intra-abdominal hemorrhage, especially the splenic hemorrhage, is a rare event in neonates (1). The incidence of splenic rupture or hemorrhage is unknown, reflecting its rarity (2). The initial symptoms of neonatal splenic rupture/hemorrhage (SRH) are non-specific which may lead to the delayed diagnosis in some cases. Despite the potential fatality, SRH can spontaneously resolve without surgical treatment. Appropriate treatment strategies for neonatal SRH remain controversial. Herein, we report the cases of two neonates with SRH and review the other cases of neonatal SRH in the English-language literature, with the aim to describe the clinical features of neonatal $\mathrm{SRH}$, to summarize therapies and outcomes, and to elucidate risk factors for mortality in neonates presenting with this rare condition.

\section{PATIENTS AND METHODS}

\section{Case Reports}

\section{Case 1}

A baby girl was delivered at term to a gravida 2, para 1 mother through cesarean section due to fetal distress and placental abruption. Her birth weight (BW) was $2,460 \mathrm{~g}$, and the 1 and 5 min Apgar scores were 6 and 7, respectively. After birth, the patient was flaccid and cyanotic. She was intubated and immediately resuscitated and with transfer to our hospital due to perinatal asphyxia.

At arrival to our hospital, the patient was pale and lethargic. Her vital signs were as follows: rectal temperature $36^{\circ} \mathrm{C}$, pulse rate, $100 / \mathrm{min}$; respiration rate $50 / \mathrm{min}$, and blood pressure, 30/10 $\mathrm{mmHg}$. She was ventilated with high frequency oscillation ventilation. Inotropic agents, blood transfusion and fluid resuscitation were administered. Laboratory data indicated metabolic acidosis. Additional laboratory parameters as follows: hemoglobin (Hb), $8.8 \mathrm{~g} / \mathrm{dL}$; the white blood cell count (WBCs), $13.9 \times 10^{3} / \mathrm{uL}$, and platelet counts $190 \times 10^{3} / \mathrm{uL}$. The prothrombin time (PT) and activated partial thromboplastin times were prolonged. The fibrinogen and D-dimer were $<50 \mathrm{mg} / \mathrm{dl}$ and $>10,000 \mathrm{FEU} \mathrm{ng} / \mathrm{mL}$. The Coombs test was negative. Cranial ultrasonography did not reveal intracranial or intraventricular hemorrhage. Perinatal asphyxia associated with maternal placental abruption and stage II hypoxic ischemic encephalopathy (HIE) were suspected. Therapeutic hypothermia was commenced at $5 \mathrm{~h}$ of age and erythropoietin was used as an adjuvant therapy. Abdominal ultrasound to investigate the cause of neonatal anemia revealed bloody ascites and splenic rupture with hemorrhage, and abdominal distension was noted during the evaluation. Emergent exploratory laparotomy and splenectomy were performed on the 1 day-old patient. During the laparostomy, massive hemoperitoneum, and splenic laceration with active bleeding were noted. Despite treatment, severe acute kidney injury, pulmonary hypertension and severe intraventricular hemorrhage occurred and was administered palliative treatment because of the expected poor outcome and high mortality.

\section{Case 2}

This 2 day-old female infant was delivered at gestational age of 39 weeks to a gravida 1 para 1 mother through vaginal delivery with a BW of 3,340 gm at an outside hospital. Apgar scores were 8 and 9 at 1 and $5 \mathrm{~min}$, respectively. The antenatal examination was unremarkable. However, she developed pallor at 2 days of age; tachycardia and decreased activity were observed as well. Laboratory examination revealed severe anemia.

Physical examination on arrival at our hospital revealed pale appearance. Her vital signs were as follows: rectal temperature of $36.3^{\circ} \mathrm{C}$; pulse rate, $143 / \mathrm{min}$; respiration rate $26 / \mathrm{min}$ and blood pressure, $76 / 54 \mathrm{mmHg}$. Laboratory examination revealed the following: $\mathrm{Hb} 5.4 \mathrm{~g} / \mathrm{dL}$; WBCs, $17.92 \times 10^{3} / \mathrm{uL}$, and platelet count $207 \times 10^{3} / \mathrm{uL}$. Abdominal ultrasonography revealed an enlarged spleen and a heterogenous mass $(4.0 \times 3.7 \times 3.8 \mathrm{~cm})$ in left upper abdomen; thus, splenic laceration with hematoma and bleeding was suspected. Abdominal computed tomography (CT) scan showed peri-splenic hematoma, $\sim 3.5 \times 3 \mathrm{~cm}$ in size, and hemoperitoneum indicating splenic laceration. After blood transfusion and fluid replace therapy, the patient stabilized and her follow-up hemoglobin level was $17.4 \mathrm{~g} / \mathrm{dL}$. She was discharged without any complications.

\section{Patient Population}

We used the keywords "neonatal splenic rupture," "neonatal splenic laceration," "neonatal splenic hemorrhage," and "neonatal splenic hematoma," to search articles in the PUBMED databases and reviewed those published articles from their inception between January 1968 and December 2019. In addition to the two cases presented herein, we identified 37 cases of neonatal SRH in the English-language literature.

\section{Data Collection and Statistical Analysis}

This study was approved by the ethics committee of Chang Gung Medical Hospital which waived the requirement for informed consent to collect anonymized data. Demographic data, precipitating factors, clinical characteristics including presenting symptoms and signs, time of onset, imaging findings, treatments, and outcomes were collected and analyzed. Preterm birth was defined as birth before 37 weeks of gestation. Low birth weight was defined as $\mathrm{BW}<2,500 \mathrm{~g}$. Age of SRH presentation was defined as the time of the emergence of first symptoms or signs after birth. Categorical variables were reported as absolute numbers and percentages and analyzed using chi-square or Fisher's exact test. Continuous variables were analyzed using parametric or non-parametric tests as appropriate. Continuous variables were reported as means with standard deviation for parametric data and as medians with interquartile range for nonparametric data. Risk factors for mortality were analyzed by univariate logistic regression and presented as odds ratios (OR) with $95 \%$ confidence intervals (CI). A $p<0.05$ was considered to indicate statistical significance. The IBM SPSS statistics package, version 21 (IBM Corp., Armonk, NY, USA) was used for all statistical analyses. 


\section{RESULTS}

There were 39 neonates with the diagnosis of SRH during the study period. The demographic data and detailed information on these 39 cases are presented in Table 1. The rates of fullterm and preterm neonates were $72 \%(28 / 39)$ and $18 \%(11 / 39)$, respectively. The median BW was $3,200(2,470-3,620) \mathrm{g}$, and four neonates were large for gestational age (LGA, BW > $4,000 \mathrm{~g})$. Additionally, 74\% (29/39) of the patients were delivered through vaginal delivery whereas the remaining patients $26 \%$ $(10 / 39)$ were delivered through cesarean section. The time of $\mathrm{SRH}$ presentation ranged between $3 \mathrm{~h}$ and 5 days of age. The precipitating factors included prolonged labor $(n=3)$, birth trauma $(n=2)$, breech $(n=1)$, wandering spleen $(n=1)$, assaulted before delivery $(n=1)$, post resuscitation $(n=5)$, congenital deficiency in coagulation factor VII deficiency $(n$ $=1)$, erythroblastosis fetalis $(n=4)$, hemophilia A $(n=3)$, family history of hemophilia A $(n=1)$, and preterm neonate $(n=11)$. Therefore, the rate of congenital coagulation disorders (congenital coagulation factor VII and hemophilia A) was 13\% in the current cohort.

The review of all reported reports revealed that the initial presenting symptoms and signs included pallor or anemia $(n=23)$, abdominal discoloration/distension $(n=23)$, tachycardia/shock $(n=10)$, scrotal hematoma or swollen ( $n$ $=7)$, lethargy $(n=5)$, vomiting $(n=4)$, poor feeding $(n$ $=3$ ), and death $(n=1)$. Abdominal ultrasonography was performed in 24 neonates; eight of these neonates were also evaluated by abdominal CT as an adjuvant diagnostic tool. Four neonates underwent abdominal CT as the initial diagnostic tool. Twenty-seven neonates (69\%) received surgical treatment; 16 neonates received splenectomy; 6 neonates received repair of the spleen rupture with tissue-glue or surgery; 1 neonate received the peritoneal flap; 1 neonate was managed with splenic autotransplantation; 3 neonates underwent exploratory laparotomy only. There were seven death in this study cohort; therefore the mortality rate was $18 \%(7 / 39)$. Case 13 was found dead at $7 \mathrm{~h}$ of age by the nurse; case 32 died at $72 \mathrm{~h}$ of age due to severe hyperkalemia with a heart rhythm disorder; case 34 died at $55 \mathrm{~h}$ of age; case 35 died at $103 \mathrm{~h}$ of age due to massive pulmonary hemorrhage with refractory hypoxemia; case 36 died at $60 \mathrm{~h}$ of age, and case 38 died at 1 month of age after withdrawal of life support. None of the remaining 32 survivors (82\%) exhibited neurologic sequelae, developmental delays or growth retardation.

Table 2 shows the clinical features associated with mortality. The mortality group had statistically significant higher rates of vaginal delivery $(p=0.041$, odds ratio $=0.144$, 95\% CI $0.024-$ 0.853 ). The age of presentation with neonatal SRH was associated with poor outcome. The odds ratio for mortality was 25.0 (95\% CI 2.514-248.575, $p=0.001$ ) in neonates presenting with SRH at $\leq 12 \mathrm{~h}$ of age compared to those presenting with SRH $>12 \mathrm{~h}$ of age. The mortality group had lower age of presentation with neonatal SRH that the median age was $3 \mathrm{~h}$ (IQR 1-7). Preterm birth, low BW, prolong labor, birth trauma, resuscitation, $1 \mathrm{~min}$ of Apgar score, $5 \mathrm{~min}$ of Apgar score, congenital coagulation disorders, hemoglobin level, or splenectomy were not statistically associated with mortality.

\section{DISCUSSION}

We herein presented an uncommon presentation of SRH (case 1) in the cooling era for perinatal HIE. Based on our experience in two cases and the analysis of available literature, presentation at $\leq 12 \mathrm{~h}$ of age was a risk factor for mortality in neonates with SRH. As a rare cause of hemoperitoneum in neonates, splenic injury is less frequently than injury to the liver or adrenal glands due to its well-protected position in the left upper abdominal quadrant (20). The triad of anemia, abdominal distension and shock has been described as typical clinical presentation of intra-abdominal hemorrhage in neonates (1). Clinicians should suspect abdominal hemorrhage if patients had unexplained anemia. Hepatic lesions (65\%) account for the most frequent causes of intra-abdominal hemorrhage, followed by adrenal lesions (15\%) and splenic lesion (10\%) (30). Hepatic laceration should be considered if the patient underwent umbilical venous catheter insertion (32). Patients had history of hypoxia before or during delivery may lead to adrenal gland hemorrhage because the increased pressure with congestion and the damaged endothelial cells $(33,34)$.

Case 1 neonate presented herein was born to a mother with placental abruption and was diagnosed as perinatal asphyxia with stage II HIE. Therapeutic hypothermia was performed as recommended (35). Abdominal ultrasonography performed based on the observed anemia and progressive abdominal distension revealed splenic rupture with hemorrhage. Case 1 neonate, who was at high mortality risk according to our review and analysis, nonetheless highlights that SRH can be a cause or a comorbid condition in neonates with perinatal HIE undergoing therapeutic hypothermia.

On our review, vaginal delivery was significantly associated with mortality which is thought increased intrathoracic pressure during vaginal delivery pushing the spleen into the abdominal cavity, and exposing it to direct splenic trauma through the birth canal during labor (29). Gruenwald et al. indicated that the intrathoracic pressure that forces the liver or spleen out of the diaphragm hallow can cause severe tension in their supported ligaments (36). Splenic rupture and splenic hemorrhage occur in two stages. The first stage is subcapsular hematoma formation, which is often asymptomatic, whereas the second stage is capsular rupture which leads to the development of acute symptoms of decompensation and the rapid onset of hypovolemic shock (30). The present study revealed that early presentation at $\leq 12 \mathrm{~h}$ of age was associated with poor prognosis. Splenic rupture/hemorrhage in neonates can present either within the first few hours of life or as late as the second week of life, following the rupture of the splenic capsule. In addition, splenic injury can range from the superficial lacerations to the parenchymal hemorrhage. Altogether, early presentation after birth might imply severe and extensive splenic injury, leading to earlier emergence of symptoms present earlier and difficult to recovery.

We also found that the most common presenting symptoms and signs of SRH were pallor or anemia, followed by abdominal discoloration/distension. In the present study, we also noted that $18 \%(7 / 39)$ of all reported cases of neonatal SRH presented with scrotal hematoma or swelling. Most of the affected neonates 
TABLE 1 | Clinical characteristics of neonates with splenic rupture.

\begin{tabular}{|c|c|c|c|c|c|c|c|c|c|c|c|c|}
\hline Year & Case & GA & BW (Kg) & $\begin{array}{l}\text { Precipitating } \\
\text { Factors }\end{array}$ & Labor & $\begin{array}{l}\text { AS } \\
\left(1^{\prime} \rightarrow 5^{\prime}\right)\end{array}$ & $\begin{array}{l}\text { Initial } \\
\text { symptoms/signs }\end{array}$ & $\begin{array}{l}\text { Presenting } \\
\text { time }\end{array}$ & Hemogram & $\begin{array}{l}\text { Diagnostic or image } \\
\text { studies }\end{array}$ & Treatment & Outcome \\
\hline 1968 & $1(3)$ & Term & 3.63 & $\begin{array}{l}\text { Perinatal asphyxia } \\
\text { Birth trauma }\end{array}$ & VD & $1 \rightarrow 1$ & $\begin{array}{l}\text { Grunting, pale, and } \\
\text { shock }\end{array}$ & $9 \mathrm{~h}$ & НCT 21 & Paracentesis & Splenectomy & Alive \\
\hline 1968 & $2(4)$ & Term & 3.75 & NA & VD & $9 \rightarrow 9$ & Tachypnea, tachycardia & $43 \mathrm{~h}$ & $\mathrm{Hb} 7.0$ & X-ray & Splenectomy & Alive; normal \\
\hline 1971 & $3(5)$ & Term & 4.2 & NA & VD & $9 \rightarrow 9$ & $\begin{array}{l}\text { Swollen and blue } \\
\text { scrotum, abdominal } \\
\text { distension }\end{array}$ & $6 \mathrm{~h}$ & $\begin{array}{l}\text { Hb } 8.0 \\
\text { PLT } 130 \mathrm{~K}\end{array}$ & $\begin{array}{l}\text { Intravenous } \\
\text { pyelography }\end{array}$ & Splenectomy & $\begin{array}{l}\text { Alive; normal follow-up } \\
\text { examination }\end{array}$ \\
\hline 1973 & $4(6)$ & 36 weeks & 1.7 & $\begin{array}{l}\text { Erythroblastosis } \\
\text { fetalis }\end{array}$ & $\mathrm{C} / \mathrm{S}$ & NA & $\begin{array}{l}\text { Hypotension, } \\
\text { abdominal distension }\end{array}$ & $6 \mathrm{~h}$ & НСТ 30 & Paracentesis & Splenic suture & Alive \\
\hline 1973 & $5(6)$ & 34 weeks & 2.0 & $\begin{array}{l}\text { Erythroblastosis } \\
\text { fetalis }\end{array}$ & $\mathrm{C} / \mathrm{S}$ & NA & $\begin{array}{l}\text { Abdominal girth } \\
\text { increased }\end{array}$ & $30 \mathrm{~h}$ & HCT 14 & Paracentesis & $\begin{array}{l}\text { Repaired by oxidized } \\
\text { cellulose }\end{array}$ & $\begin{array}{l}\text { Died on the second } \\
\text { postoperative day }\end{array}$ \\
\hline 1975 & $6(7)$ & 36 weeks & 3.08 & $\begin{array}{l}\text { Erythroblastosis } \\
\text { fetalis }\end{array}$ & VD & $4 \rightarrow 9$ & Anemia & $48 \mathrm{~h}$ & $\mathrm{Hb} 7.2$ & Paracentesis & Splenectomy & Alive \\
\hline 1980 & $7(8)$ & 40 weeks & 3.975 & NA & VD & $9 \rightarrow 10$ & Pale, tachypnea, shock & NA & $\mathrm{Hb} 6.4$ & Paracentesis & Exploratory laparotomy & Alive \\
\hline 1984 & $8(9)$ & Term & NA & $\begin{array}{l}\text { Mother took } \\
\text { luminal }\end{array}$ & VD & NA & $\begin{array}{l}\text { Lethargy, pale, and } \\
\text { abdominal distension }\end{array}$ & $24 \mathrm{~h}$ & $\begin{array}{l}\text { HCT } 19.6 \\
\text { PLT } 850 K\end{array}$ & X-ray: free fluid & Exploratory laparotomy & Alive; normal \\
\hline 1993 & $9(10)$ & Term & 3.05 & NA & VD & $9 \rightarrow 10$ & $\begin{array}{l}\text { Scrotal sac dilatation, } \\
\text { bilateral hematoceles, } \\
\text { hematoma in the left } \\
\text { inguinal canal, } \\
\text { hematoma of the left } \\
\text { anterior abdominal wall }\end{array}$ & $24 \mathrm{~h}$ & Normal & US & Exploratory laparotomy & $\begin{array}{l}\text { Alive; Normal follow-up } \\
\text { examinations at } 4 \text { and } \\
6 \text { months of age }\end{array}$ \\
\hline 1993 & $10(10)$ & Term & 3.2 & Prolonged labor & VD & $7 \rightarrow 10$ & $\begin{array}{l}\text { Hematoma of the left } \\
\text { scrotum and inguinal } \\
\text { canal, abdominal } \\
\text { distension }\end{array}$ & $16 \mathrm{~h}$ & $\begin{array}{l}\text { Hb:5.9 } \\
\text { HCT 20\% }\end{array}$ & US & $\begin{array}{l}\text { Splenic } \\
\text { auto-transplantation }\end{array}$ & $\begin{array}{l}\text { Alive; Normal follow-up } \\
\text { examinations }\end{array}$ \\
\hline 1999 & $11(11)$ & 36 weeks & 2.9 & $\begin{array}{l}\text { Hemophilia A } \\
\text { Preterm }\end{array}$ & NA & $8 \rightarrow 9$ & $\begin{array}{l}\text { Pale and lethargy, } \\
\text { swollen right scrotum }\end{array}$ & 5 days & HCT $6.2 \%$ & US & Splenectomy & $\begin{array}{l}\text { Alive, discharge on } \\
\text { day14 }\end{array}$ \\
\hline 2000 & $12(12)$ & 39 weeks & 4.167 & Prolonged labor & VD & $8 \rightarrow 9$ & $\begin{array}{l}\text { Pale, grunting, lethargy, } \\
\text { abdominal distention }\end{array}$ & 2 days & $\begin{array}{l}\text { HCT 16\% } \\
\text { PLT 198K }\end{array}$ & X-ray: Unremarkable & Splenectomy & $\begin{array}{l}\text { Alive; discharged on } \\
\text { postoperative day } 8\end{array}$ \\
\hline 2000 & $13(13)$ & Term & 2.82 & Birth trauma & VD & $8 \rightarrow 10$ & $\begin{array}{l}\text { Death was noted by } \\
\text { nurse }\end{array}$ & $7 \mathrm{~h}$ & NA & Autopsy & Non & Dead at $7 \mathrm{~h}$ old \\
\hline 2000 & $14(14)$ & 26 weeks & 0.746 & $\begin{array}{l}\text { Prolonged labor } \\
\text { Preterm } \\
\text { Resuscitation }\end{array}$ & VD & $0 \rightarrow 1$ & $\begin{array}{l}\text { Hypotension, tense and } \\
\text { discolored abdomen }\end{array}$ & $36 \mathrm{~h}$ & Hb 11.6 & US & $\begin{array}{l}\text { Packed the site with } \\
\text { surgical and } \\
\text { 2-component fibrin } \\
\text { sealant }\end{array}$ & Alive \\
\hline 2000 & $15(1)$ & Term & 3.6 & Prolonged labor & VD & $8 \rightarrow 9$ & $\begin{array}{l}\text { Abdominal distension, } \\
\text { pale looking }\end{array}$ & $<5 \mathrm{~h}$ & $\mathrm{Hb} 4.3$ & СT & Blood transfusion & $\begin{array}{l}\text { Alive; discharged at } \\
\text { day } 10\end{array}$ \\
\hline 2002 & $16(2)$ & 38 weeks & 3.55 & NA & VD & $8 \rightarrow 9$ & $\begin{array}{l}\text { Coffee ground emesis, } \\
\text { abdominal distension, } \\
\text { pale }\end{array}$ & $16 \mathrm{~h}$ & $\mathrm{Hb} 8.5$ & Paracentesis, US & $\begin{array}{l}\text { Packed with gel-foam } \\
\text { and surgical }\end{array}$ & $\begin{array}{l}\text { Alive, discharged at } \\
\text { day } 22\end{array}$ \\
\hline 2003 & $17(15)$ & Term & 5.08 & NA & VD & $8 \rightarrow 9$ & $\begin{array}{l}\text { Right hemiscrotal } \\
\text { swelling and bluish } \\
\text { discoloration }\end{array}$ & $4 \mathrm{~h}$ & $\mathrm{Hb} 10.6$ & US, CT & Blood transfusion & Alive \\
\hline
\end{tabular}


TABLE 1 | Continued

\begin{tabular}{|c|c|c|c|c|c|c|c|c|c|c|c|c|}
\hline Year & Case & GA & BW (Kg) & $\begin{array}{l}\text { Precipitating } \\
\text { Factors }\end{array}$ & Labor & $\begin{array}{l}\text { AS } \\
\left(1^{\prime} \rightarrow 5^{\prime}\right)\end{array}$ & $\begin{array}{l}\text { Initial } \\
\text { symptoms/signs }\end{array}$ & $\begin{array}{l}\text { Presenting } \\
\text { time }\end{array}$ & Hemogram & $\begin{array}{l}\text { Diagnostic or image } \\
\text { studies }\end{array}$ & Treatment & Outcome \\
\hline 2004 & $18(16)$ & Term & 3.6 & NA & VD & $8 \rightarrow 9$ & $\begin{array}{l}\text { Bilious vomiting, pale, } \\
\text { abdominal distension }\end{array}$ & 2 days & $\mathrm{Hb} 5.8$ & CT & Conservative treatment & $\begin{array}{l}\text { Alive, discharged at } \\
\text { hospital } 12\end{array}$ \\
\hline 2004 & $19(17)$ & 36 weeks & 2.62 & $\begin{array}{l}\text { Wandering spleen } \\
\text { Preterm }\end{array}$ & $\mathrm{C} / \mathrm{S}$ & $8 \rightarrow 10$ & $\begin{array}{l}\text { Intense mucous and } \\
\text { cutaneous paleness, } \\
\text { scant motility, } \\
\text { hypotonia, ecchymosis } \\
\text { in the right side of the } \\
\text { scrotum }\end{array}$ & $22 \mathrm{~h}$ & $\begin{array}{l}\text { Hb } 5.5 \\
\text { HCT 16\% } \\
\text { PLT 155K }\end{array}$ & US, CT & Peritoneal flap & $\begin{array}{l}\text { Alive; normal at the age } \\
\text { of } 1 \text { year old }\end{array}$ \\
\hline 2006 & $20(18)$ & 27 weeks & 0.845 & $\begin{array}{l}\text { Breech } \\
\text { Preterm }\end{array}$ & VD & $6 \rightarrow 8$ & $\begin{array}{l}\text { Apnea, sudden } \\
\text { hypotension, pallor, } \\
\text { poor perfusion, } \\
\text { abdominal distension }\end{array}$ & $20 \mathrm{~h}$ & $\mathrm{Hb} 6.4$ & Non & $\begin{array}{l}\text { argon beam and tissue } \\
\text { glue }\end{array}$ & $\begin{array}{l}\text { Alive; normal at the age } \\
\text { of } 11 \text { months }\end{array}$ \\
\hline 2007 & $21(19)$ & 36 weeks & 2.3 & $\begin{array}{l}\text { Splenic cavernous } \\
\text { hemangioma }\end{array}$ & VD & NA & Hypovolemia, anemia & $48 \mathrm{~h}$ & $\mathrm{Hb} 8.5$ & US, CT & Splenectomy & Alive \\
\hline 2008 & $22(20)$ & Term & 3.2 & Non & VD & NA & $\begin{array}{l}\text { Progressive pallor, } \\
\text { abdominal distension, } \\
\text { poor feeding, bruising } \\
\text { on the left side of neck, } \\
\text { chest and both soles }\end{array}$ & $30 \mathrm{~h}$ & НCT 18.9 & US, CT & Conservative treatment & $\begin{array}{l}\text { Alive; discharged at } 15 \\
\text { days of age; resolution } \\
\text { of hematoma at } 1 \\
\text { month old }\end{array}$ \\
\hline 2010 & $23(21)$ & Term & 3.452 & Hemophilia A & VD & $9 \rightarrow 9$ & $\begin{array}{l}\text { lethargy and pale with a } \\
\text { weak cry }\end{array}$ & $72 \mathrm{~h}$ & $\mathrm{Hb} 5.0$ & CT & Splenectomy & Alive \\
\hline 2011 & $24(22)$ & Term & 3.64 & $\begin{array}{l}\text { Assaulted } 2 \text { weeks } \\
\text { before delivery }\end{array}$ & VD & NA & $\begin{array}{l}\text { Abdominal distension, } \\
\text { subconjunctival } \\
\text { hemorrhages, facial } \\
\text { bruising, pallor, poor } \\
\text { feeding }\end{array}$ & $12 \mathrm{~h}$ & $\begin{array}{l}\text { Hb } 9.5 \\
\text { HCT31\% } \\
\text { PLT 351K }\end{array}$ & $\begin{array}{l}\text { US, Needle } \\
\text { paracentesis }\end{array}$ & Splenectomy & Alive \\
\hline 2011 & $25(23)$ & 35 weeks & 2.48 & Erythroblastosis & $\mathrm{C} / \mathrm{S}$ & $8 \rightarrow 9$ & Abdominal distension & 3 days & $\mathrm{Hb} 5.6$ & Paracentesis, US & $\begin{array}{l}\text { Peritoneal drainage, } \\
\text { blood transfusion }\end{array}$ & Alive \\
\hline 2012 & $26(24)$ & Term & 3.45 & $\begin{array}{l}\text { Family history of } \\
\text { Hemophilia A }\end{array}$ & VD & $9 \rightarrow 9$ & $\begin{array}{l}\text { Pallor, lethargy, poor } \\
\text { feeding and vomiting }\end{array}$ & 3 days & HCT $14 \%$ & CT & Splenectomy & $\begin{array}{l}\text { Alive; discharge on } \\
\text { POD } 31\end{array}$ \\
\hline 2015 & $27(25)$ & Term & 3.5 & NA & VD & NA & $\begin{array}{l}\text { Swollen, discolored, } \\
\text { and tender left } \\
\text { hemi-scrotum }\end{array}$ & 2 days & $\mathrm{Hb} 7.1$ & US, CT & Splenectomy & Alive \\
\hline 2016 & $28(26)$ & 40 weeks & 2.385 & $\begin{array}{l}\text { Splenic } \\
\text { hemangioma }\end{array}$ & VD & NA & $\begin{array}{l}\text { Pale, vomiting, tender } \\
\text { abdomen }\end{array}$ & $24 \mathrm{~h}$ & NA & US & Splenectomy & Alive \\
\hline 2017 & $29(27)$ & 39 weeks & 4.2 & $\begin{array}{l}\text { Congenital } \\
\text { deficiency in } \\
\text { coagulation factor } \\
\text { VII }\end{array}$ & VD & $9 \rightarrow 10$ & $\begin{array}{l}\text { Pale, grunting, nasal } \\
\text { flaring and respiratory } \\
\text { distress, extensive } \\
\text { parietal } \\
\text { cephalohematoma, } \\
\text { abdominal } \\
\text { enlargement, } \\
\text { abdominal tenderness }\end{array}$ & $24 \mathrm{~h}$ & $\begin{array}{l}\mathrm{Hb} 4.0 \\
\text { HCT } 14 \%\end{array}$ & US & Splenectomy & $\begin{array}{l}\text { Alive; self-limiting } \\
\text { coagulation at } 1 \text { year } \\
\text { old }\end{array}$ \\
\hline
\end{tabular}


TABLE 1 | Continued

\begin{tabular}{|c|c|c|c|c|c|c|c|c|c|c|c|c|}
\hline Year & Case & GA & BW (Kg) & $\begin{array}{l}\text { Precipitating } \\
\text { Factors }\end{array}$ & Labor & $\begin{array}{l}\text { AS } \\
\left(1^{\prime} \rightarrow 5^{\prime}\right)\end{array}$ & $\begin{array}{l}\text { Initial } \\
\text { symptoms/signs }\end{array}$ & $\begin{array}{l}\text { Presenting } \\
\text { time }\end{array}$ & Hemogram & $\begin{array}{l}\text { Diagnostic or image } \\
\text { studies }\end{array}$ & Treatment & Outcome \\
\hline 2017 & $30(28)$ & 24 weeks & 0.71 & $\begin{array}{l}\text { Preterm } \\
\text { Resuscitation }\end{array}$ & $\mathrm{C} / \mathrm{S}$ & $6 \rightarrow 8$ & Hemoglobin dropped & $8 \mathrm{~h}$ & $\mathrm{Hb} 8.7$ & US, CT & $\begin{array}{l}\text { Massive blood } \\
\text { transfusion (FFP, LPR) }\end{array}$ & $\begin{array}{l}\text { Alive; resolution of } \\
\text { splenic hematoma }\end{array}$ \\
\hline 2017 & $31(29)$ & 40 weeks & 2.95 & Hemophilia A & VD & Normal & Jaundice and pallor & 4 days & $\mathrm{Hb} 6.9$ & US, CT & Factor VIII infusions & $\begin{array}{l}\text { Alive; discharge at } 18 \\
\text { days old; normal } \\
\text { growth and } \\
\text { development }\end{array}$ \\
\hline 2017 & $32(30)$ & 32 weeks & 1.96 & Preterm & $\mathrm{C} / \mathrm{S}$ & $3 \rightarrow 3$ & $\begin{array}{l}\text { Diffuse cutaneous } \\
\text { hematomas, bleeding } \\
\text { at the puncture points, } \\
\text { and bleeding at the } \\
\text { umbilical venous } \\
\text { catheter }\end{array}$ & $3 \mathrm{~h}$ & Hb 4.4 & US & $\begin{array}{l}\text { Massive blood } \\
\text { transfusion }\end{array}$ & $\begin{array}{l}\text { Died at } 72 \mathrm{~h} \text { due to } \\
\text { major hyperkalemia } \\
\text { with heart rhythm } \\
\text { disorders }\end{array}$ \\
\hline 2017 & $33(30)$ & 38 weeks & 3.56 & $\begin{array}{l}\text { Broncho- } \\
\text { aspiration and } \\
\text { then ventilated } \\
\text { with neopuff }\end{array}$ & VD & $3 \rightarrow 5$ & $\begin{array}{l}\text { Pallor, respiratory } \\
\text { distress, abdominal } \\
\text { bloating, tachycardia }\end{array}$ & $12 \mathrm{~h}$ & $\mathrm{Hb} 6.4$ & US & Splenectomy & Alive; Hurler's disease \\
\hline 2017 & $34(30)$ & Term & 3.145 & NA & VD & $7 \rightarrow 8$ & $\begin{array}{l}\text { Cutaneous-mucosal } \\
\text { pallor, respiratory } \\
\text { distress, a bloated } \\
\text { abdomen and } \\
\text { hypotonia }\end{array}$ & $1 \mathrm{~h}$ & Hb 10.3 & US & Splenectomy & Died at $55 \mathrm{~h}$ \\
\hline 2017 & $35(30)$ & Term & NA & Suction extraction & VD & $2 \rightarrow 4$ & $\begin{array}{l}\text { Pallor, shock, } \\
\text { abdominal distension }\end{array}$ & $1 \mathrm{~h}$ & Hb 10.3 & US & Splenectomy & Died at $103 \mathrm{~h}$ \\
\hline 2017 & $36(30)$ & 40 weeks & 2.88 & NA & $\mathrm{C} / \mathrm{S}$ & $1 \rightarrow 6$ & $\begin{array}{l}\text { Pale, abdominal } \\
\text { distension }\end{array}$ & $1 \mathrm{~h}$ & Hb 10.6 & US & Hemostatic mesh & Died at $60 \mathrm{~h}$ \\
\hline 2018 & $37(31)$ & Term & 3.885 & Non & VD & $9 \rightarrow 9$ & $\begin{array}{l}\text { Bluish discoloration, } \\
\text { hypotension, marked } \\
\text { abdominal distention }\end{array}$ & $6 \mathrm{~h}$ & $\mathrm{Hb} 5.5$ & US & Blood transfusion & Alive; Normal \\
\hline $\begin{array}{l}\text { Our } \\
\text { case }\end{array}$ & 38 & Term & 2.46 & Resuscitation & $\mathrm{C} / \mathrm{S}$ & $6 \rightarrow 7$ & $\begin{array}{l}\text { Less urine output, } \\
\text { hypovolemic shock, } \\
\text { abdominal distension }\end{array}$ & $6 \mathrm{~h}$ & $\begin{array}{l}\mathrm{Hb} 8.8 \\
\text { PLT } 190 \mathrm{~K}\end{array}$ & US & Splenectomy & Dead at 1 month old \\
\hline $\begin{array}{l}\text { Our } \\
\text { case }\end{array}$ & 39 & 39 weeks & 3.34 & Non & VD & $8 \rightarrow 9$ & $\begin{array}{l}\text { Tachycardia, pale } \\
\text { appearance, } \\
\text { deteriorating activity }\end{array}$ & 2 days & $\begin{array}{l}\text { Hb } 5.8 \\
\text { PLT } 207 \mathrm{~K}\end{array}$ & US, CT & Blood transfusion & $\begin{array}{l}\text { Alive; Normal follow-up } \\
\text { examinations }\end{array}$ \\
\hline
\end{tabular}

GA, gestational age; BW, birth weight; NA, unknown; VD, vaginal delivery; C/S, cesarean section; AS, Apgar scores; US, ultrasonography; CT, computed tomography; POD, post-operative day; (\#) indicates reference number. 
TABLE 2 | Risk factors associated with mortality in neonates with splenic rupture/hemorrhage.

\begin{tabular}{|c|c|c|c|c|c|c|}
\hline & All & Survival & Mortality & $p$-value & Odds ratio & $95 \% \mathrm{Cl}$ \\
\hline \multicolumn{7}{|l|}{ Categorical variable } \\
\hline Patient number ( $n, \%)$ & 39 & $32(82)$ & $7(18)$ & & & \\
\hline Preterm (n, \%) & 11 & $9(82)$ & $2(18)$ & 0.981 & 1.022 & $0.167-6.258$ \\
\hline Prolong labor (n, \%) & 5 & $4(80)$ & $1(20)$ & 0.898 & 1.167 & $0.11-12.381$ \\
\hline $\operatorname{VD}(n, \%)$ & 29 & $26(90)$ & $3(10)$ & ${ }^{*} 0.041$ & 0.144 & $0.024-0.853$ \\
\hline Birth trauma $(n, \%)$ & 3 & $1(33)$ & $2(67)$ & 0.077 & 12.4 & $0.94-163.581$ \\
\hline Resuscitation (n, \%) & 5 & $3(60)$ & $2(40)$ & 0.169 & 3.867 & $0.51-29.304$ \\
\hline Age of presentation < $12 \mathrm{~h}(n, \%)$ & 12 & $6(50)$ & $6(50)$ & *0.001 & 25.0 & $2.514-248.575$ \\
\hline Splenectomy (n, \%) & 14 & $11(79)$ & $3(21)$ & 0.966 & 0.964 & $0.185-5.030$ \\
\hline Congenital coagulation disorder ( $n, \%)$ & 5 & $5(100)$ & 0 & 0.263 & NS & NS \\
\hline \multicolumn{7}{|l|}{ Continuous variable } \\
\hline Age of presentation (IQR) & $23(6-48)$ & $24(12-24)$ & $3(1-7)$ & ${ }^{\star} 0.044$ & 0.879 & $0.775-0.997$ \\
\hline Birth weight (kg) (IQR) & $3.2(2.47-3.62)$ & $3.45(2.55-3.63)$ & $2.82(2.0-3.145)$ & 0.193 & 0.562 & $0.236-1.338$ \\
\hline Apgar score 1 min (IQR) & $8(6-9)$ & $8(6-9)$ & $4(2-7)$ & 0.054 & 0.734 & $0.536-1.006$ \\
\hline Apgar score 5 min (IQR) & $9(8,9)$ & $9(9)$ & $7(4-9)$ & 0.114 & 0.773 & $0.561-1.064$ \\
\hline Hemoglobin (g/dl) (IQR) & $6.7(5.5-8.8)$ & $6.4(5.5-7.5)$ & $10.3(6.6-10.5)$ & 0.073 & 1.583 & $0.958-2.617$ \\
\hline
\end{tabular}

NS, non-specific.

(74\%) were born through vaginal delivery, and four out of them (14\%) were LGA neonates. Higher birth weight is not a precipitating factor leading to SRH. Regarding precipitating and risk factors, most cases of neonatal SRH were idiopathic. However, previous studies reported that splenic rupture occurs in those with an abnormal spleen such as those with erythroblastosis $(6,7,37)$. Congenital coagulation disorders accounted for $13 \%(5 / 39)$ of SRH of all neonatal SRH cases in the present study cohort. Therefore, investigation for congenital coagulation disorders is recommended in neonates with SRH.

Based on our literature review, the prognosis was comparable between patients who received non-surgical treatment and those who underwent splenectomy. The first case of neonatal SRH who recovered after surgical treatment was reported by Rogers in 1934 (38). Splenectomy has been considered as the first line of treatment for neonatal SRH in the past decade (36), and the preservation of the spleen is desirable (39). Recent studies recommend non-surgical management as the first-line of therapy for SRH due to the risk of overwhelming post splenectomy sepsis $(14,17,40)$. Studies also indicated that children are at higher risk of post-splenectomy sepsis compared with adults $(40,41)$. In addition, advances in ultrasound technology allow the definitive diagnosis of causes underlying intra-abdominal hemorrhage such as renal and splenic hemorrhage $(42,43)$. Computed tomography to evaluate the severity of splenic trauma may also provide safe non-surgical management in these patients (44). With recent improvement in diagnostic imaging and alternative approaches, splenectomy is no longer a priority. Our literature review reveals that long-term outcome is favorable in neonates who survive SRH.

The present study has several limitations. First, the small sample size of the study might have affected the statistical analysis. However, neonatal SRH is extremely rare and the current study included the largest cohort in statistical analyses.
Additionally, the retrospective study designs might have introduced bias.

\section{CONCLUSION}

The mortality of neonatal splenic rupture/hemorrhage was $18 \%$ in this study. Vaginal delivery and the presentation at $\leq 12 \mathrm{~h}$ of age were risk factors for mortality. Investigation for congenital coagulation disorders is recommended in neonate with SRH.

\section{DATA AVAILABILITY STATEMENT}

The raw data supporting the conclusions of this article will be made available by the authors, without undue reservation.

\section{ETHICS STATEMENT}

The studies involving human participants were reviewed and approved by the ethics committee of Chang Gung Medical Hospital. Written informed consent for participation was not provided by the participants' legal guardians/next of kin because: this retrospective study was approved by the ethics committee of Chang Gung Medical Hospital and waived the requirement to obtain informed consent of collecting anonymized data.

\section{AUTHOR CONTRIBUTIONS}

H-PC conceived the study, collected information from the literature, and drafted the manuscript. R-HF and J-JL provided professional guidance and manuscript modification. M-CC conceived the study, provided professional guidance, and manuscript modification. All authors contributed to the article and approved the submitted version. 


\section{REFERENCES}

1. Bickler S, Ramachandran V, Gittes GK, Alonso M, Snyder LC. Nonoperative management of newborn splenic injury: a case report. J Pediatr Surg. (2000) 35:500-1. doi: 10.1016/S0022-3468(00)90222-7

2. Hui C, Tsui K. Splenic rupture in a newborn. J Pediatr Surg. (2002) 37:1-3. doi: 10.1053/jpsu.2002.31641

3. Sokol DM, Tompkins D, Izant JR Jr. Rupture of the spleen and liver in the newborn: a report of the first survivor and a review of the literature. J Pediatr Surg. (1974) 9:227-9. doi: 10.1016/S0022-3468(74)80127-2

4. Delta BG, Eisenstein EM, Rothenberg MA. Rupture of a normal spleen in the newborn: report of a survival and review of the literature. Clin Pediatr. (1968) 7:373-6. doi: 10.1177/000992286800700620

5. Leape LL, Bordy DM. Neonatal rupture of the spleen: report of a case successfully treated after spontaneous cessation of hemorrhage. Pediatrics. (1971) 47:101-4.

6. Simmons MA, Burrington JD, Wayne ER, Hathaway EW. Splenic rupture in neonates with erythroblastosis fetalis. Am J Dis Child. (1973) 126:679-81. doi: 10.1001/archpedi.1973.02110190547018

7. Coulter J, Raine P. Rupture of the spleen in erythroblastosis fetalis. Arch Dis Child. (1975) 50:398. doi: 10.1136/adc.50.5.398

8. Chryss C, Aaron SW. Successful treatment of rupture of normal spleen in newborn. Am J Dis Child. (1980) 134:418-9. doi: 10.1001/archpedi.1980.04490010072021

9. Traggis D, Maunz D, Baroudy R. Splenic hemorrhage in a neonate of a mother on anticonvulsant therapy. J Pediatr Surg. (1984) 19:598-9. doi: 10.1016/S0022-3468(84)80114-1

10. Bader D, Mogilner JG, Berger A, Eldar S, Reich D, Siplovich L. Neonatal splenic rupture: an unusual manifestation. Pediatr Surg Int. (1993) 8:151-3. doi: $10.1007 / \mathrm{BF} 00184225$

11. Johnson-Robbins LA, Porter JC, Horgan JM. Splenic rupture in a newborn with hemophilia a: case report and revew of the literature. Clin Pediatr. (1999) 38:117-9. doi: 10.1177/000992289903800210

12. Longobardi Y, Lessin MS, Kleinman M, Wesselhoeft CW, Berns DS. Unsuspected splenic rupture in a neonate. Pediatr Emerg Care. (2000) 16:2830. doi: 10.1097/00006565-200002000-00009

13. Acar K, Cinbis M. A neonatal death due to rupture of the normal spleen. Int J Pediatr Neonatol. (2000) 2:4928. doi: 10.5580/76d

14. Moisiuk SE, Scott S, Davi M, Wiseman N. Salvage of the ruptured spleen in an infant with very low birth weight. J Pediatr Surg. (2000) 35:1822-3. doi: 10.1053/jpsu.2000.19269

15. Perdomo Y, Fiore N, Reyna T. Splenic injury presenting with isolated scrotal findings in a stable newborn. J Pediatr Surg. (2003) 38:1673-5. doi: 10.1016/S0022-3468(03)00581-5

16. Driscoll K, Benjamin LC, Gilbert JC, Chahine AA. Nonoperative management of neonatal splenic rupture. Am Surg. (2004) 70:1085-7.

17. Balliu P-R, Bregante J, Pérez-Velasco M-C, Fiol M, Galiana C, Herrera $\mathrm{M}$, et al. Splenic haemorrhage in a newborn as the first manifestation of wandering spleen syndrome. J Pediatr Surg. (2004) 39:240-2. doi: 10.1016/j.jpedsurg.2003.10.025

18. Ting J, Lam B, Ngai C, Leung W, Chan K. Splenic rupture in a premature neonate. Hong Kong Med J. (2006) 12:68-70.

19. Pachl M, Elmalik K, Cohen M, Kamupira S, Walker J, Murthi G. Ruptured splenic cavernous hemangioma in a neonate. J Pediatr Surg. (2008) 43:407-9. doi: 10.1016/j.jpedsurg.2007.09.080

20. Lewis L, Sanoj K, Poojari G, Kamath PS. Neonate subcapsular splenic hematoma. Indian J Pediatr. (2008) 75:950-2. doi: 10.1007/s12098-0080199-y

21. Tengsupakul S, Sedrak A, Freed J, Martinez-Carde L, Fireman F, Pierre L, et al. Splenic rupture in a newborn with severe hemophiliacase report and review. J Pediatr Hematol Oncol. (2010) 32:323-6. doi: 10.1097/MPH.0b013e3181d640ae

22. Lloyd LG, de Witt W. Splenic rupture in a neonate-a rare complication. South Afr J Child Health. (2011) 5:94-6. doi: 10.1007/s13193-011-0064-8

23. Serce O, Topcuoglu S, Kurt G, Karatekin G. Splenic rupture in neonate with hemolytic anemia. Int J Med Med Sci. (2011) 3:236-8.

24. Adamu I, Asarian A, Xiao P. Splenic rupture and intracranial haemorrhage in a haemophilic neonate: case report and literature review. Afr J Paediatr Surg. (2012) 9:163. doi: 10.4103/0189-6725.99408
25. Tiboni S, Abdulmajid U, Pooboni S, Wighton C, Eradi B, Dagash H. Spontaneous splenic hemorrhage in the newborn. Eur J Pediatr Surg Rep. (2015) 3:71-3. doi: 10.1055/s-0035-1564610

26. Martinez-Leo B, Vidal-Medina J, Cervantes-Ledezma J, de Leon-Rivera AD, Diaz-Velasco E. Spontaneous rupture of splenic hemangioma in a neonate. $J$ Neonatal Surg. (2016) 5:36. doi: 10.21699/jns.v5i3.365

27. Zeid MA, Zeid CA, Noun P, Yacoub A, Loutfi G. Neonatal spleen rupture, shock, and factor VII deficiency. Am Acad Pediatr. (2018) 142:130. doi: 10.1542/peds.142.1_MeetingAbstract.13

28. Joshi S, Mulinge I, Kamat M. An extremely premature neonate with severe anemia. J Neonatal Surg. (2017) 6:33. doi: 10.21699/jns.v6i2.547

29. Badawy SM, Rossoff J, Yallapragada S, Liem RI, Sharathkumar AA. Successful medical management of a neonate with spontaneous splenic rupture and severe hemophilia. Hematol Oncol A Stem Cell Ther. (2017) 10:29-32. doi: 10.1016/j.hemonc.2016.04.001

30. Descamps C-S, Cneude F, Hays S, Rayet I, Piolat C, Epiard C, et al. Early hypovolemic shock and abdominal distention due to neonatal splenic rupture: urgency of diagnosis and management. Eur J Pediatr. (2017) 176:1245-50. doi: 10.1007/s00431-017-2968-y

31. Moreira A, Das H. Acute life-threatening hemorrhage in neonates with severe hemophilia a: a report of 3 cases. J Investig Med High Impact Case Rep. (2018) 6:2324709618800349. doi: 10.1177/2324709618800349

32. Pignotti MS, Monciotti F, Frati P, Fineschi V. Hepatic laceration due to umbilical venous catheter malpositioning. Pediatr Neonatol. (2017) 58:386-7. doi: 10.1016/j.pedneo.2016.09.008

33. Wang $\mathrm{CH}$, Chen SJ, Yang LY, Tang RB. Neonatal adrenal hemorrhage presenting as a multiloculated cystic mass. J Chin Med Assoc. (2008) 71:481-4. doi: 10.1016/S1726-4901(08)70153-9

34. Koplewitz BZ, Daneman A, Cutz E, Hellmann J. Neonatal adrenal congestion: a sonographic-pathologic correlation. Pediatr Radiol. (1998) 28:958-62. doi: 10.1007/s002470050509

35. Chiang M-C, Jong Y-J, Lin C-H. Therapeutic hypothermia for neonates with hypoxic ischemic encephalopathy. Pediatr Neonatol. (2017) 58:475-83. doi: 10.1016/j.pedneo.2016.11.001

36. Gruenwald P. Rupture of liver and spleen in the newborn infant. J Pediatr. (1948) 33:195-201. doi: 10.1016/S0022-3476(48)80057-0

37. O'Neill EA, O'Brian ER, Hyun HB. Rupture of the spleen in the newborn infant: report of a survival. JAMA. (1965) 193:959-60. doi: 10.1001/jama.1965.03090110097031

38. Rogers G. Hemoperitoneum resulting from hepatic birth traumatism. Am J Obstetr Gynecol. (1934) 27:841-50. doi: 10.1016/S0002-9378(34)91050-4

39. Buess E, Illi O, Soder C, Hanimann B. Ruptured spleen in children-15year evolution intherapeutic concepts. Eur J Pediatr Surg. (1992) 2:157-61. doi: 10.1055/s-2008-1063429

40. Hoefnagel R. Susceptibility to infection after splenectomy performed in childhood. In: Clinical Proceedings-Children's Hospital of the District of Columbia. (1956). p. 48.

41. Lynch AM, Kapila R. Overwhelming postsplenectomy infection. Infect Dis Clin N Am. (1996) 10:693-707. doi: 10.1016/S0891-5520(05)70322-6

42. Blaivas M, Quinn J. Diagnosis of spontaneous splenic rupture with emergency ultrasonography. Ann Emerg Med. (1998) 32:627-30. doi: 10.1016/S0196-0644(98)70046-0

43. Newman B, Smith S. Unusual renal mass in a newborn infant. Radiology. (1987) 163:193-4. doi: 10.1148/radiology.163.1.3547494

44. Williams RA, Black JJ, Sinow RM, Wilson ES. Computed tomographyassisted management of splenic trauma. Am J Surg. (1997) 174:276-9. doi: 10.1016/S0002-9610(97)00135-9

Conflict of Interest: The authors declare that the research was conducted in the absence of any commercial or financial relationships that could be construed as a potential conflict of interest.

Copyright (c) 2021 Chang, Fu, Lin and Chiang. This is an open-access article distributed under the terms of the Creative Commons Attribution License (CC BY). The use, distribution or reproduction in other forums is permitted, provided the original author(s) and the copyright owner(s) are credited and that the original publication in this journal is cited, in accordance with accepted academic practice. No use, distribution or reproduction is permitted which does not comply with these terms. 